\title{
On Efficient Method for System of Fractional Differential Equations
}

\author{
Najeeb Alam Khan, ${ }^{1}$ Muhammad Jamil, ${ }^{2,}{ }^{3}$ Asmat Ara, ${ }^{1}$ \\ and Nasir-Uddin Khan ${ }^{1}$ \\ ${ }^{1}$ Department of Mathematics, University of Karachi, Karachi 75270, Pakistan \\ ${ }^{2}$ Abdul Salam School of Mathematical Sciences, GC University, Lahore, Pakistan \\ ${ }^{3}$ Department of Mathematics, NEDUET, Karachi 75270, Pakistan
}

Correspondence should be addressed to Najeeb Alam Khan, njbalam@yahoo.com

Received 14 December 2010; Accepted 5 February 2011

Academic Editor: J. J. Trujillo

Copyright (c) 2011 Najeeb Alam Khan et al. This is an open access article distributed under the Creative Commons Attribution License, which permits unrestricted use, distribution, and reproduction in any medium, provided the original work is properly cited.

The present study introduces a new version of homotopy perturbation method for the solution of system of fractional-order differential equations. In this approach, the solution is considered as a Taylor series expansion that converges rapidly to the nonlinear problem. The systems include fractional-order stiff system, the fractional-order Genesio system, and the fractional-order matrix Riccati-type differential equation. The new approximate analytical procedure depends only on two components. Comparing the methodology with some known techniques shows that the present method is relatively easy, less computational, and highly accurate.

\section{Introduction}

Fractional differential equations have received considerable interest in recent years and have been extensively investigated and applied for many real problems which are modeled in different areas. One possible explanation of such unpopularity could be that there are multiple nonequivalent definitions of fractional derivatives [1]. Another difficulty is that fractional derivatives have no evident geometrical interpretation because of their nonlocal character. However, during the last 12 years fractional calculus starts to attract much more attention of scientists. It was found that various, especially interdisciplinary, applications [26] can be elegantly modeled with the help of the fractional derivatives.

The homotopy perturbation method is a powerful devise for solving nonlinear problems. This method was introduced by He [7-9] in the year 1998. In this method, the solution is considered as the summation of an infinite series that converges rapidly. 
This technique is used for solving nonlinear chemical engineering equations [10], timefractional Swift-Hohenberg (S-H) equation [11], viscous fluid flow equation [12], FourthOrder Integro-Differential equations [13], nonlinear dispersive $K(m, n, 1)$ equations [14], Long Porous Slider equation [15], and Navier-Stokes equations [16]. It can be said that He's homotopy perturbation method is a universal one, which is able to solve various kinds of nonlinear equations. The new homotopy perturbation method (NHPM) was applied to linear and nonlinear ODEs [17].

In this paper, we construct the solution of system of fractional-order differential equations by extending the idea of $[17,18]$. This method leads to computable and efficient solutions to linear and nonlinear operator equations. The corresponding solutions of the integer-order equations are found to follow as special cases of those of fractional-order equations.

We consider the system of fractional-order equations of the form

$$
D^{\alpha_{i}} y_{i}(t)+F_{i}\left(t, y_{1}, y_{2}, y_{3}, \ldots, y_{n}\right)=f_{i}(t), \quad y_{i}\left(t_{0}\right)=c_{i}, \quad 0<\alpha_{i} \leq 1, i=1,2, \ldots, n
$$

\section{Basic Definitions}

We give some basic definitions, notations, and properties of the fractional calculus theory used in this work.

Definition 2.1. The Riemann-Liouville fractional integral operator $J^{\mu}$ of order $\mu$ on the usual Lebesgue space $L_{1}[a, b]$ is given by

$$
\begin{gathered}
J^{\mu} f(x)=\frac{1}{\Gamma(\mu)} \int_{0}^{x}(x-t)^{\mu-1} f(t) d t, \quad \mu>0, \\
J^{0} f(x)=f(x) .
\end{gathered}
$$

It has the following properties:

(i) $J^{\mu}$ exists for any $x \in[a, b]$,

(ii) $J^{\mu} J^{\beta}=J^{\mu+\beta}$,

(iii) $J^{\mu} J^{\beta}=J^{\beta} J^{\mu}$,

(iv) $J^{\alpha} J^{\beta} f(x)=J^{\beta} J^{\alpha} f(x)$,

(v) $J^{\mu}(x-a)^{\gamma}=(\Gamma(\gamma+1) / \Gamma(\alpha+\gamma+1))(x-a)^{\mu+\gamma}$,

where $f \in L_{1}[a, b], \mu, \beta \geq 0$ and $\gamma>-1$.

Definition 2.2. The Caputo definition of fractal derivative operator is given by

$$
D^{\mu} f(x)=J^{m-\mu} D^{n} f(x)=\frac{1}{\Gamma(m-\mu)} \int_{0}^{t}(x-\tau)^{m-\mu-1} f^{(m)}(\tau) d \tau,
$$


where $(m-1<\mu \leq m, m \in N, x>0)$. It has the following two basic properties for $m-1<$ $\mu \leq m$ and $f \in L_{1}[a, b]$ :

$$
\begin{gathered}
D^{\mu} J^{\mu} f(x)=f(x), \\
J^{\mu} D^{\mu} f(x)=f(x)-\sum_{k=0}^{m-1} f^{(k)}\left(0^{+}\right) \frac{(x-a)^{k}}{k !}, \quad x>0 .
\end{gathered}
$$

\section{Analysis of New Homotopy Perturbation Method}

Let us consider the system of nonlinear differential equations

$$
\mathscr{A}_{i}\left(y_{i}\right)=f_{i}(t), \quad t \in \Omega,
$$

where $\mathcal{A}_{i}$ are the operators, $f_{i}$ are known functions and $y_{i}$ are sought functions. Assume that operators $\mathcal{A}_{i}$ can be written as

$$
\mathcal{A}_{i}\left(y_{i}\right)=\mathcal{L}_{i}\left(y_{i}\right)+\mathcal{N}_{i}\left(y_{i}\right)
$$

where $\mathcal{L}_{i}$ are the linear operators and $\mathcal{N}_{i}$ are the nonlinear operators. Hence, (3.1) can be rewritten as follows:

$$
\mathcal{L}_{i}\left(y_{i}\right)+\mathcal{N}_{i}\left(y_{i}\right)=f_{i}(t), \quad t \in \Omega
$$

We define the operators $\mathscr{\ell}_{i}$ as

$$
\mathscr{L}_{i}\left(Y_{i} ; p\right) \equiv(1-p)\left(\mathcal{L}_{i}\left(Y_{i}\right)-\mathcal{L}_{i}\left(y_{i, 0}\right)\right)+p\left(\mathcal{A}_{i}(Y)-f_{i}\right),
$$

where $p \in[0,1]$ is an embedding or homotopy parameter, $Y_{i}(t ; p): \Omega \times[0,1] \rightarrow \Re$ and $y_{i, 0}$ are the initial approximation of solution of the problem in (3.3) can be written as

$$
\mathscr{L}_{i}\left(Y_{i} ; p\right) \equiv \mathcal{L}_{i}\left(Y_{i}\right)-\mathcal{L}_{i}\left(y_{i, 0}\right)+p \mathcal{L}_{i}\left(y_{i, 0}\right)+p\left(\mathcal{N}_{i}\left(Y_{i}\right)-f_{i}\right)
$$

Clearly, the operator equations $\mathscr{L}_{i}(v, 0)=0$ and $\mathscr{L}_{i}(v, 1)=0$ are equivalent to the equations $\mathcal{L}_{i}\left(Y_{i}\right)-\mathcal{L}\left(y_{i, 0}\right)=0$ and $\mathcal{A}_{i}(Y)-f_{i}(t)=0$, respectively. Thus, a monotonous change of parameter $p$ from zero to one corresponds to a continuous change of the trivial problem $\mathcal{L}_{i}\left(Y_{i}\right)-\mathcal{L}_{i}\left(y_{i, 0}\right)=0$ to the original problem. Operator $\mathscr{\ell}_{i}\left(Y_{i}, p\right)$ is called a homotopy map. Next, we assume that the solution of equation $\mathscr{L}_{i}\left(Y_{i}, p\right)$ can be written as a power series in embedding parameter $p$, as follows:

$$
Y_{i}=Y_{i, 0}+p Y_{i, 1}, \quad i=1,2,3, \ldots, n
$$

Now, let us write (3.5) in the following form:

$$
\mathcal{L}_{i}\left(Y_{i}\right)=y_{i, 0}(t)+p\left(f_{i}-\mathcal{N}_{i}\left(Y_{i}\right)-y_{i, 0}(t)\right) .
$$


By applying the inverse operator, $\mathcal{L}_{i}^{-1}$ to both sides of (3.7), we have

$$
Y_{i}=\mathcal{L}_{i}^{-1} y_{i, 0}(t)+p\left(\mathcal{L}_{i}^{-1} f-\mathcal{L}_{i}^{-1} \mathcal{N}_{i}\left(Y_{i}\right)-\mathcal{L}_{i}^{-1} y_{i, 0}(t)\right)
$$

Suppose that the initial approximation of (3.3) has the form

$$
y_{i, 0}(t)=\sum_{n=0}^{\infty} a_{i, n} P_{n}(t), \quad i=1,2,3, \ldots, n,
$$

where $a_{i, n}, n=0,1,2, \ldots$ are unknown coefficients and $P_{n}(t), n=0,1,2, \ldots$ are specific functions on the problem. By substituting (3.6) and (3.9) into (3.8), we get

$$
Y_{i, 0}+p Y_{i, 1}=\mathcal{L}_{i}^{-1}\left(\sum_{n=0}^{\infty} a_{i, n} P_{n}(t)\right)+p\left(\mathcal{L}_{i}^{-1} f_{i}-\mathcal{L}_{i}^{-1} \mathcal{N}_{i}\left(Y_{i, 0}+p Y_{i, 1}\right)-\mathcal{L}_{i}^{-1}\left(\sum_{n=0}^{\infty} a_{i, n} P_{n}(t)\right)\right) .
$$

Equating the coefficients of like powers of $p$, we get the following set of equations:

$$
\begin{gathered}
\text { coefficient of } p^{0}: \Upsilon_{0}=\mathcal{L}^{-1}\left(\sum_{n=0}^{\infty} a_{i, n} P_{n}(t)\right) \\
\text { coefficient of } p^{1}: \Upsilon_{1}=\mathcal{L}_{i}^{-1}\left(f_{i}\right)+\mathcal{L}_{i}^{-1}\left(Y_{i, 1}\right)-\mathcal{L}_{i}^{-1} \mathcal{N}_{i}\left(Y_{i, 0}\right)
\end{gathered}
$$

Now, we solve these equations in such a way that $Y_{i, 1}(t)=0$. Therefore, the approximate solution may be obtained as

$$
y_{i}(t)=Y_{i, 0}(t)=\mathcal{L}^{-1}\left(\sum_{n=0}^{\infty} a_{i, n} P_{n}(t)\right)
$$

\section{Applications}

\section{Application 1}

Consider the following linear fractional-order 2-by-2 stiff system:

$$
\begin{aligned}
& D_{t}^{\alpha} u(t)=k(-1-\varepsilon) u(t)+k(1-\varepsilon) v(t), \\
& D_{t}^{\alpha} v(t)=k(1-\varepsilon) u(t)+k(-1-\varepsilon) v(t)
\end{aligned}
$$

with the initial conditions

$$
u(0)=1, \quad v(0)=3,
$$


where $k$ and $\varepsilon$ are constants. To obtain the solution of (4.1) by NHPM, we construct the following homotopy:

$$
\begin{aligned}
& (1-p)\left(D_{t}^{\alpha} U(t)-u_{0}(t)\right)+p\left(D_{t}^{\alpha} U(t)-k(-1-\varepsilon) U(t)-k(1-\varepsilon) V(t)\right)=0, \\
& (1-p)\left(D_{t}^{\alpha} V(t)-v_{0}(t)\right)+p\left(D_{t}^{\alpha} V(t)-k(1-\varepsilon) U(t)-k(-1-\varepsilon) V(t)\right)=0 .
\end{aligned}
$$

Applying the inverse operator, $J_{t}^{\alpha}$ of $D_{t}^{\alpha}$ both sides of the above equation, we obtain

$$
\begin{aligned}
& U(t)=U(0)+J_{t}^{\alpha} u_{0}(t)-p J_{t}^{\alpha}\left(u_{0}(t)-k(-1-\varepsilon) U(t)-k(1-\varepsilon) V(t)\right) \\
& V(t)=V(0)+J_{t}^{\alpha} v_{0}(t)-p J_{t}^{\alpha}\left(v_{0}(t)-k(1-\varepsilon) U(t)-k(-1-\varepsilon) V(t)\right) .
\end{aligned}
$$

The solution of (4.1) to has the following form:

$$
U(t)=U_{0}(t)+p U_{1}(t), \quad V(t)=V_{0}(t)+p V_{1}(t)
$$

Substituting (4.5) in (4.4) and equating the coefficients of like powers of $p$, we get the following set of equations:

$$
\begin{aligned}
& U_{0}(t)=U(0)+J_{t}^{\alpha} u_{0}(t), \quad V_{0}(t)=V(0)+J_{t}^{\alpha} v_{0}(t), \\
& U_{1}(t)=J_{t}^{\alpha}\left(-u_{0}(t)+k(-1-\varepsilon) U_{0}(t)+k(1-\varepsilon) V_{0}(t)\right), \\
& V_{1}(t)=J_{t}^{\alpha}\left(-v_{0}(t)+k(1-\varepsilon) U_{0}(t)+k(-1-\varepsilon) V_{0}(t)\right) .
\end{aligned}
$$

Assuming $u_{0}(t)=\sum_{n=0}^{20} a_{n} P_{n}, v_{0}(t)=\sum_{n=0}^{20} b_{n} P_{n}, P_{k}=t^{k}, U(0)=u(0)$, and $V(0)=v(0)$ and solving the above equation for $U_{1}(t)$ and $V_{1}(t)$ lead to the result

$$
\begin{aligned}
& U_{1}(t)=\frac{\left(2 k-4 \varepsilon k-a_{0}\right) t^{\alpha}}{\Gamma(\alpha+1)}-\frac{a_{1} t^{\alpha+1}}{\Gamma(\alpha+2)}-\frac{2 a_{2} t^{\alpha+2}}{\Gamma(\alpha+3)}-\frac{6 a_{3} t^{\alpha+3}}{\Gamma(\alpha+4)}-\frac{24 a_{4} t^{2 \alpha}}{\Gamma(\alpha+5)}+\cdots \\
& V_{1}(t)=\frac{\left(-2 k-4 \varepsilon k-b_{0}\right) t^{\alpha}}{\Gamma(\alpha+1)}-\frac{b_{1} t^{\alpha+1}}{\Gamma(\alpha+2)}-\frac{2 b_{2} t^{\alpha+2}}{\Gamma(\alpha+3)}-\frac{6 b_{3} t^{\alpha+3}}{\Gamma(\alpha+4)}-\frac{24 b_{4} t^{2 \alpha}}{\Gamma(\alpha+5)}+\cdots
\end{aligned}
$$

Vanishing $U_{1}(t)$ and $V_{1}(t)$ lets the coefficients $a_{i}, b_{i}, i=0,1,2, \ldots$ by taking $\alpha=1$ the following values:

$$
\begin{array}{ccc}
a_{0}=2 k(1-2 \varepsilon), & a_{1}=-4 k^{2}\left(1-2 \varepsilon^{2}\right), & a_{2}=4 k^{3}\left(1-2 \varepsilon^{3}\right), \\
a_{3}=\frac{-8 k^{4}\left(1-2 \varepsilon^{4}\right)}{3}, & a_{4}=\frac{4 k^{5}\left(1-2 \varepsilon^{5}\right)}{3}, & a_{5}=\frac{-8 k^{6}\left(1-2 \varepsilon^{6}\right)}{15}, \\
a_{6}=\frac{8 k^{7}\left(1-2 \varepsilon^{7}\right)}{45}, & a_{7}=\frac{-16 k^{8}\left(1-2 \varepsilon^{8}\right)}{315}, & a_{8}=\frac{4 k^{9}\left(1-2 \varepsilon^{9}\right)}{315}, \\
a_{9}=\frac{-8 k^{10}\left(1-2 \varepsilon^{10}\right)}{2835}, & a_{10}=\frac{8 k^{11}\left(1-2 \varepsilon^{11}\right)}{14175}, & a_{11}=\frac{-16 k^{12}\left(1-2 \varepsilon^{12}\right)}{155925},
\end{array}
$$




$$
\begin{array}{ccr}
a_{12}=\frac{8 k^{13}\left(1-2 \varepsilon^{13}\right)}{467775}, & a_{13}=\frac{-16 k^{14}\left(1-2 \varepsilon^{14}\right)}{6081075}, & a_{14}=\frac{16 k^{15}\left(1-2 \varepsilon^{15}\right)}{42567525} \\
a_{15}=\frac{-16 k^{16}\left(1-2 \varepsilon^{16}\right)}{155925}, & a_{16}=\frac{4 k^{17}\left(1-2 \varepsilon^{17}\right)}{638512875}, & a_{17}=\frac{-8 k^{18}\left(1-2 \varepsilon^{18}\right)}{10854718875} \\
a_{18}=\frac{8 k^{19}\left(1-2 \varepsilon^{19}\right)}{97692469875}, & a_{19}=\frac{-16 k^{20}\left(1-2 \varepsilon^{20}\right)}{1856156927625}, & a_{20}=\frac{8 k^{21}\left(1-2 \varepsilon^{21}\right)}{9280784638125} \\
b_{0}=-2 k(1+2 \varepsilon), & b_{1}=4 k^{2}\left(1+2 \varepsilon^{2}\right), & b_{2}=-4 k^{3}\left(1+2 \varepsilon^{3}\right) \\
b_{3}=\frac{8 k^{4}\left(1+2 \varepsilon^{4}\right)}{3}, & b_{4}=\frac{-4 k^{5}\left(1+2 \varepsilon^{5}\right)}{3}, & b_{5}=\frac{8 k^{6}\left(1+2 \varepsilon^{6}\right)}{15}, \\
b_{6}=\frac{-8 k^{7}\left(1+2 \varepsilon^{7}\right)}{45}, & b_{7}=\frac{16 k^{8}\left(1+2 \varepsilon^{8}\right)}{315}, & b_{8}=\frac{-4 k^{9}\left(1+2 \varepsilon^{9}\right)}{315} \\
b_{9}=\frac{8 k^{10}\left(1+2 \varepsilon^{10}\right)}{2835}, & b_{10}=\frac{-8 k^{11}\left(1+2 \varepsilon^{11}\right)}{14175}, & b_{11}=\frac{16 k^{12}\left(1+2 \varepsilon^{12}\right)}{155925} \\
b_{12}=\frac{-8 k^{13}\left(1+2 \varepsilon^{13}\right)}{467775}, & b_{13}=\frac{16 k^{14}\left(1+2 \varepsilon^{14}\right)}{6081075}, & b_{14}=\frac{-16 k^{15}\left(1+2 \varepsilon^{15}\right)}{42567525}, \\
b_{15}=\frac{16 k^{16}\left(1+2 \varepsilon^{16}\right)}{155925}, & b_{16}=\frac{-4 k^{17}\left(1+2 \varepsilon^{17}\right)}{638512875}, & b_{17}=\frac{8 k^{18}\left(1+2 \varepsilon^{18}\right)}{10854718875} \\
b_{18}=\frac{-8 k^{19}\left(1+2 \varepsilon^{19}\right)}{97692469875}, & b_{19}=\frac{16 k^{20}\left(1+2 \varepsilon^{20}\right)}{1856156927625}, & b_{20}=\frac{-8 k^{21}\left(1+2 \varepsilon^{21}\right)}{9280784638125}
\end{array}
$$

Therefore, we obtain the solutions of (4.1) as

$$
\begin{aligned}
& u(t)=1+\frac{2 k(1-2 \varepsilon) t^{\alpha}}{\Gamma(\alpha+1)}-\frac{4 k^{2}\left(1-2 \varepsilon^{2}\right) t^{\alpha+1}}{\Gamma(\alpha+2)}+\frac{8 k^{3}\left(1-2 \varepsilon^{3}\right) t^{\alpha+2}}{\Gamma(\alpha+3)}-\frac{16 k^{4}\left(1-2 \varepsilon^{4}\right) t^{\alpha+3}}{\Gamma(\alpha+4)}+\cdots, \\
& v(t)=3-\frac{2 k(1+2 \varepsilon) t^{\alpha}}{\Gamma(\alpha+1)}+\frac{4 k^{2}\left(1+2 \varepsilon^{2}\right) t^{\alpha+1}}{\Gamma(\alpha+2)}-\frac{8 k^{3}\left(1+2 \varepsilon^{3}\right) t^{\alpha+2}}{\Gamma(\alpha+3)}-\frac{16 k^{4}\left(1+2 \varepsilon^{4}\right) t^{\alpha+3}}{\Gamma(\alpha+4)}+\cdots
\end{aligned}
$$

Our aim is to study the mathematical behavior of the solution $u(t)$ and $v(t)$ for different values of $\alpha$. This goal can be achieved by forming Pade' approximants, which have the advantage of manipulating the polynomial approximation into a rational function to gain more information about $u(t)$ and $v(t)$. It is well known that Pade' approximants will converge on the entire real axis, if $u(t)$ and $v(t)$ are free of singularities on the real axis. It is of interest to note that Pade' approximants give results with no greater error bounds than approximation by polynomials. To consider the behavior of solution for different values of $\alpha$, we will take advantage of the explicit formula (4.9) available for $0<\alpha \leq 1$ and consider the following two special cases. 
Case 1. Setting $\alpha=1, k=50, \varepsilon=0.01$ in (4.9), we obtain the approximate solution in a series form as

$$
\begin{aligned}
& u_{[10,11]}(t)=\frac{1+148.73 t+1203.65 t^{2}+51963.1 t^{3}+\cdots}{1+50.7628 t+1227.89 t^{2}+18726.5 t^{3}+\cdots} \\
& v_{[10,11]}(t)=\frac{3+69.439 t+3823.59 t^{2}+40311.9 t^{3}+\cdots}{1+57.1463 t+1550.5 t^{2}+26447.2 t^{3}+\cdots}
\end{aligned}
$$

Case 2. In this case, we will examine the linear fractional stiff equation (4.1). Setting $\alpha=$ $1 / 2, k=50, \varepsilon=0.01$ in (4.9) gives

$$
\begin{aligned}
& u(t)=1+\frac{196 t^{1 / 2}}{\sqrt{\pi}}-\frac{39992 t^{3 / 2}}{3 \sqrt{\pi}}+\frac{7999984 t^{5 / 2}}{15 \sqrt{\pi}}-\frac{228571424 t^{7 / 2}}{15 \sqrt{\pi}}+\cdots, \\
& v(t)=3-\frac{204 t^{1 / 2}}{\sqrt{\pi}}+\frac{13336 t^{3 / 2}}{\sqrt{\pi}}-\frac{2666672 t^{5 / 2}}{5 \sqrt{\pi}}+\frac{533333344 t^{7 / 2}}{35 \sqrt{\pi}}-\cdots .
\end{aligned}
$$

For simplicity, let $t^{1 / 2}=z$, then

$$
\begin{aligned}
& u(z)=1+\frac{196 z}{\sqrt{\pi}}-\frac{39992 z^{3}}{3 \sqrt{\pi}}+\frac{7999984 z^{5}}{15 \sqrt{\pi}}-\frac{228571424 z^{7}}{15 \sqrt{\pi}}+\cdots \\
& v(z)=3-\frac{204 z}{\sqrt{\pi}}+\frac{13336 z^{3}}{\sqrt{\pi}}-\frac{2666672 z^{5}}{5 \sqrt{\pi}}+\frac{533333344 z^{7}}{35 \sqrt{\pi}}-\cdots
\end{aligned}
$$

Calculating the [10/11] Pade' approximants and recalling that $z=t^{1 / 2}$, we get

$$
\begin{gathered}
u_{[10,11]}=\frac{9.58 \times 10^{-8}+0.0000126 t^{1 / 2}+0.0002357 t-0.0001051 t^{3 / 2}-\cdots}{9.581 \times 10^{-8}+2.0904 \times 10^{-6} t^{1 / 2}+4.56399 \times 10^{-6} t+0.000096 t^{2}+\cdots} \\
v_{[10,11]}=\frac{2.66 \times 10^{123}-1.216 \times 10^{125} t^{1 / 2}+8.69947 \times 10^{125} t+\cdots}{8.88 \times 10^{122}-6.45605 \times 10^{123} t^{1 / 2}+4.22967 \times 10^{124} t+\cdots}
\end{gathered}
$$

\section{Application 2}

Consider the following nonlinear fractional-order 2-by-2 stiff system:

$$
\begin{gathered}
D_{t}^{\alpha} u(t)=-1002 u(t)+1000 v^{2}(t), \\
D_{t}^{\alpha} v(t)=u(t)-v(t)-v^{2}(t)
\end{gathered}
$$

with the initial conditions

$$
u(0)=1, \quad v(0)=1 .
$$


To obtain the solution of (4.14) by NHPM, we construct the following homotopy:

$$
\begin{gathered}
(1-p)\left(D_{t}^{\alpha} U(t)-u_{0}(t)\right)+p\left(D_{t}^{\alpha} U(t)+1002 U(t)-1000 V^{2}(t)\right)=0, \\
(1-p)\left(D_{t}^{\alpha} V(t)-v_{0}(t)\right)+p\left(D_{t}^{\alpha} V(t)-U(t)+V(t)+V^{2}(t)\right)=0 .
\end{gathered}
$$

Applying the inverse operator, $J_{t}^{\alpha}$ of $D_{t}^{\alpha}$ both sides of the above equation, we obtain

$$
\begin{gathered}
U(t)=U(0)+J_{t}^{\alpha} u_{0}(t)-p J_{t}^{\alpha}\left(u_{0}(t)+1002 U(t)-1000 V^{2}(t)\right) \\
V(t)=V(0)+J_{t}^{\alpha} v_{0}(t)-p J_{t}^{\alpha}\left(v_{0}(t)-U(t)+V(t)+V^{2}(t)\right)
\end{gathered}
$$

The solution of (4.14) to have the following form:

$$
U(t)=U_{0}(t)+p U_{1}(t), \quad V(t)=V_{0}(t)+p V_{1}(t)
$$

Substituting (4.18) in (4.17) and equating the coefficients of like powers of $p$, we get the following set of equations:

$$
\begin{gathered}
U_{0}(t)=U(0)+J_{t}^{\alpha} u_{0}(t), \quad V_{0}(t)=V(0)+J_{t}^{\alpha} v_{0}(t), \\
U_{1}(t)=J_{t}^{\alpha}\left(-u_{0}(t)-1002 U_{0}(t)+1000 V_{0}^{2}(t)\right) \\
V_{1}(t)=J_{t}^{\alpha}\left(-v_{0}(t)+U_{0}(t)-V_{0}(t)-V_{0}^{2}(t)\right)
\end{gathered}
$$

Assuming $u_{0}(t)=\sum_{n=0}^{20} a_{n} P_{n}, v_{0}(t)=\sum_{n=0}^{20} b_{n} P_{n}, P_{k}=t^{k}, U(0)=u(0)$, and $V(0)=v(0)$ and solving the above equation for $U_{1}(t)$ and $V_{1}(t)$ lead to the result

$$
\begin{aligned}
& U_{1}(t)=\frac{-\left(a_{0}+2\right) t^{\alpha}}{\Gamma(\alpha+1)}-\frac{a_{1} t^{\alpha+1}}{\Gamma(\alpha+2)}-\frac{2 a_{2} t^{\alpha+2}}{\Gamma(\alpha+3)}-\frac{6 a_{3} t^{\alpha+3}}{\Gamma(\alpha+4)}-\frac{24 a_{4} t^{\alpha+4}}{\Gamma(\alpha+5)}-\cdots, \\
& V_{1}(t)=\frac{-\left(b_{0}+1\right) t^{\alpha}}{\Gamma(\alpha+1)}-\frac{b_{1} t^{\alpha+1}}{\Gamma(\alpha+2)}-\frac{2 b_{2} t^{\alpha+2}}{\Gamma(\alpha+3)}-\frac{6 b_{3} t^{\alpha+3}}{\Gamma(\alpha+4)}-\frac{24 b_{4} t^{\alpha+4}}{\Gamma(\alpha+5)}-\cdots .
\end{aligned}
$$

Vanishing $U_{1}(t)$ and $V_{1}(t)$ lets the coefficients $a_{i}, b_{i}, i=0,1,2, \ldots$ to take the following values:

$$
\begin{gathered}
a_{0}=-2, a_{1}=4, a_{2}=-4, a_{3}=\frac{8}{3}, a_{4}=\frac{-4}{3}, \ldots, a_{20}=\frac{-8}{9280784638125}, \\
b_{0}=-1, b_{1}=1, b_{2}=\frac{-1}{2}, b_{3}=\frac{1}{6}, b_{4}=\frac{-1}{24}, \ldots, b_{20}=\frac{-1}{2432902008176640000} .
\end{gathered}
$$


Therefore, we obtain the solution of (4.14) as

$$
\begin{aligned}
& u(t)=1-\frac{2 t^{\alpha}}{\Gamma(\alpha+1)}+\frac{4 t^{\alpha+1}}{\Gamma(\alpha+2)}-\frac{8 t^{\alpha+2}}{\Gamma(\alpha+3)}+\frac{16 t^{\alpha+3}}{\Gamma(\alpha+4)}-\frac{32 t^{\alpha+4}}{\Gamma(\alpha+5)}+\cdots \\
& v(t)=1-\frac{t^{\alpha}}{\Gamma(\alpha+1)}+\frac{t^{\alpha+1}}{\Gamma(\alpha+2)}-\frac{t^{\alpha+2}}{\Gamma(\alpha+3)}+\frac{t^{\alpha+3}}{\Gamma(\alpha+4)}-\frac{t^{\alpha+4}}{\Gamma(\alpha+5)}+\cdots
\end{aligned}
$$

The exact solution of (4.14) for $\alpha=1$ is $u(t)=e^{-2 t}, v(t)=e^{-t}$.

\section{Application 3}

Consider the following nonlinear Genesio system with fractional derivative:

$$
\begin{gathered}
D_{t}^{\alpha} u(t)=v(t), \\
D_{t}^{\alpha} v(t)=w(t) \\
D_{t}^{\alpha} w(t)=-c u(t)-b v(t)-a w(t)+u^{2}(t)
\end{gathered}
$$

with the initial conditions

$$
u(0)=0.2, \quad v(0)=-0.3, \quad w(0)=0.1,
$$

where $a, b$, and $c$ are constants. To obtain the solution of (4.23) by NHPM, we construct the following homotopy:

$$
\begin{gathered}
(1-p)\left(D_{t}^{\alpha} U(t)-u_{0}(t)\right)+p\left(D_{t}^{\alpha} U(t)-V(t)\right)=0 \\
(1-p)\left(D_{t}^{\alpha} V(t)-v_{0}(t)\right)+p\left(D_{t}^{\alpha} V(t)-W(t)\right)=0 \\
(1-p)\left(D_{t}^{\alpha} W(t)-w_{0}(t)\right)+p\left(D_{t}^{\alpha} W(t)+c U(t)+b V(t)+a W(t)-U^{2}(t)\right)=0 .
\end{gathered}
$$

Applying the inverse operator, $J_{t}^{\alpha}$ of $D_{t}^{\alpha}$ both sides of the above equation, we obtain

$$
\begin{gathered}
U(t)=U(0)+J_{t}^{\alpha} u_{0}(t)-p J_{t}^{\alpha}\left(u_{0}(t)-V(t)\right), \\
V(t)=V(0)+J_{t}^{\alpha} v_{0}(t)-p J_{t}^{\alpha}\left(v_{0}(t)-W(t)\right), \\
W(t)=W(t)+J_{t}^{\alpha} w_{0}(t)-p J_{t}^{\alpha}\left(w_{0}(t)+c U(t)+b V(t)+a W(t)-U^{2}(t)\right) .
\end{gathered}
$$

The solution of (4.23) to have the following form:

$$
U(t)=U_{0}(t)+p U_{1}(t), \quad V(t)=V_{0}(t)+p V_{1}(t), \quad W(t)=W_{0}(t)+p W_{1}(t)
$$


Substituting (4.27) in (4.26) and equating the coefficients of like powers of $p$, we get the following set of equations:

$$
\begin{gathered}
U_{0}(t)=U(0)+J_{t}^{\alpha} u_{0}(t), \quad V_{0}(t)=V(0)+J_{t}^{\alpha} v_{0}(t), \quad W_{0}(t)=W(0)+J_{t}^{\alpha} w_{0}(t), \\
U_{1}(t)=J_{t}^{\alpha}\left(-u_{0}(t)+V_{0}(t)\right), \\
V_{1}(t)=J_{t}^{\alpha}\left(-v_{0}(t)+W_{0}(t)\right), \\
W_{1}(t)=J_{t}^{\alpha}\left(-w_{0}(t)-c U_{0}(t)-b V_{0}(t)-a V_{0}(t)+W_{0}^{2}(t)\right) .
\end{gathered}
$$

Assuming $u_{0}(t)=\sum_{n=0}^{20} a_{n} P_{n}, v_{0}(t)=\sum_{n=0}^{20} b_{n} P_{n}, w_{0}(t)=\sum_{n=0}^{20} c_{n} P_{n}, P_{k}=t^{k}, U(0)=u(0)$, $V(0)=v(0), W(0)=w(0), a=1.2, b=2.92$, and $c=6$, and solving the above equation for $U_{1}(t), V_{1}(t)$ and $W_{1}(t)$ lead to the result

$$
\begin{gathered}
U_{1}(t)=\frac{-\left(a_{0}+(3 / 10)\right) t^{\alpha}}{\Gamma(\alpha+1)}-\frac{a_{1} t^{\alpha+1}}{\Gamma(\alpha+2)}-\frac{2 a_{2} t^{\alpha+2}}{\Gamma(\alpha+3)}-\frac{6 a_{3} t^{\alpha+3}}{\Gamma(\alpha+4)}-\frac{24 a_{4} t^{\alpha+4}}{\Gamma(\alpha+5)}-\cdots, \\
V_{1}(t)=\frac{\left(b_{0}-(1 / 10)\right) t^{\alpha}}{\Gamma(\alpha+1)}-\frac{b_{1} t^{\alpha+1}}{\Gamma(\alpha+2)}-\frac{2 b_{2} t^{\alpha+2}}{\Gamma(\alpha+3)}-\frac{6 b_{3} t^{\alpha+3}}{\Gamma(\alpha+4)}-\frac{24 b_{4} t^{\alpha+4}}{\Gamma(\alpha+5)}-\cdots, \\
W_{1}(t)=\frac{-\left(c_{0}+(101 / 250)\right) t^{\alpha}}{\Gamma(\alpha+1)}-\frac{c_{1} t^{\alpha+1}}{\Gamma(\alpha+2)}-\frac{2 c_{2} t^{\alpha+2}}{\Gamma(\alpha+3)}-\frac{6 c_{3} t^{\alpha+3}}{\Gamma(\alpha+4)}-\frac{24 c_{4} t^{\alpha+4}}{\Gamma(\alpha+5)}-\cdots
\end{gathered}
$$

Vanishing $U_{1}(t), V_{1}(t)$, and $W_{1}(t)$ lets the coefficients $a_{i}, b_{i}, c_{i}, i=0,1,2, \ldots$ to take the following values:

$$
\begin{gathered}
a_{0}=\frac{-3}{10}, \quad a_{1}=\frac{1}{10}, \quad a_{2}=\frac{-101}{500}, \quad a_{3}=\frac{2341}{7500}, \quad a_{4}=\frac{-377}{6250}, \ldots, \\
a_{20}=\frac{-64170831419403533391899}{1160098079765625000000000000000000}, \\
b_{0}=\frac{1}{10}, \quad b_{1}=\frac{-101}{250}, \quad b_{2}=\frac{2341}{2500}, \quad b_{3}=\frac{-754}{3125}, \quad b_{4}=\frac{-5153}{75000}, \ldots, \\
b_{20}=\frac{338555437777297749556491}{89238313828125000000000000000000}, \\
c_{0}=\frac{-101}{250}, \quad c_{1}=\frac{23411}{1250}, \quad c_{2}=\frac{-2262}{3125}, \quad c_{3}=\frac{-5153}{75000}, \quad c_{4}=\frac{-508141}{3750000}, \ldots, \\
c_{20}=\frac{5838803330656480870609733}{19334967996093750000000000000000000} .
\end{gathered}
$$


Therefore, we obtain the solutions of (4.23) as

$$
\begin{aligned}
& u(t)=\frac{1}{5}-\frac{3 t^{\alpha}}{10 \Gamma(\alpha+1)}+\frac{t^{\alpha+1}}{10 \Gamma(\alpha+2)}-\frac{101 t^{\alpha+2}}{250 \Gamma(\alpha+3)}+\frac{2341 t^{\alpha+3}}{1250 \Gamma(\alpha+4)}-\frac{4524 t^{\alpha+4}}{3125 \Gamma(\alpha+5)}+\cdots \\
& v(t)=\frac{-3}{10}+\frac{t^{\alpha}}{10 \Gamma(\alpha+1)}-\frac{101 t^{\alpha+1}}{250 \Gamma(\alpha+2)}+\frac{2341 t^{\alpha+2}}{1250 \Gamma(\alpha+3)}-\frac{4524 t^{\alpha+3}}{3125 \Gamma(\alpha+4)}-\frac{5153 t^{\alpha+4}}{3125 \Gamma(\alpha+5)}+\cdots \\
& w(t)=\frac{1}{10}-\frac{101 t^{\alpha}}{250 \Gamma(\alpha+1)}+\frac{2341 t^{\alpha+1}}{1250 \Gamma(\alpha+2)}-\frac{4524 t^{\alpha+2}}{3125 \Gamma(\alpha+3)}-\frac{5153 t^{\alpha+3}}{3125 \Gamma(\alpha+4)}-\frac{508141 t^{\alpha+4}}{156250 \Gamma(\alpha+5)}+\cdots
\end{aligned}
$$

\section{Application 4}

Finally, we consider the following nonlinear matrix Riccati differential equation with fractional derivative:

$$
D_{t}^{\alpha} Y(t)=-Y^{2}(t)+Q, \quad Y(0)=0,
$$

where $Q=(1 / 2)\left[\begin{array}{cc}1 & -1 \\ 1 & 1\end{array}\right]\left[\begin{array}{cc}1 & 0 \\ 0 & 100\end{array}\right]\left[\begin{array}{cc}1 & 1 \\ -1 & 1\end{array}\right]$. To find the solution of this equation by NHPM, we will treat the matrix equation as a system of fractional-order differential equations

$$
\begin{gathered}
D_{t}^{\alpha} u(t)=-u^{2}(t)-v(t) z(t)+\frac{101}{2}, \\
D_{t}^{\alpha} v(t)=-u(t) v(t)-v(t) w(t)-\frac{99}{2}, \\
D_{t}^{\alpha} z(t)=-u(t) v(t)-v(t) w(t)-\frac{99}{2}, \\
D_{t}^{\alpha} w(t)=-u^{2}(t)-v(t) z(t)+\frac{101}{2},
\end{gathered}
$$

with the initial conditions

$$
u(0)=0, \quad v(0)=0, \quad z(0)=0, \quad w(0)=0 .
$$

Therefore, we obtain the solution of (4.33) as

$$
\begin{aligned}
& u(t)=w(t)=\frac{1}{5}-\frac{3 t^{\alpha}}{10 \Gamma(\alpha+1)}+\frac{t^{\alpha+1}}{10 \Gamma(\alpha+2)}-\frac{101 t^{\alpha+2}}{250 \Gamma(\alpha+3)}+\frac{2341 t^{\alpha+3}}{1250 \Gamma(\alpha+4)}-\frac{4524 t^{\alpha+4}}{3125 \Gamma(\alpha+5)}+\cdots \\
& v(t)=z(t)=\frac{-3}{10}+\frac{t^{\alpha}}{10 \Gamma(\alpha+1)}-\frac{101 t^{\alpha+1}}{250 \Gamma(\alpha+2)}+\frac{2341 t^{\alpha+2}}{1250 \Gamma(\alpha+3)}-\frac{4524 t^{\alpha+3}}{3125 \Gamma(\alpha+4)}-\frac{5153 t^{\alpha+4}}{3125 \Gamma(\alpha+5)}+\cdots
\end{aligned}
$$




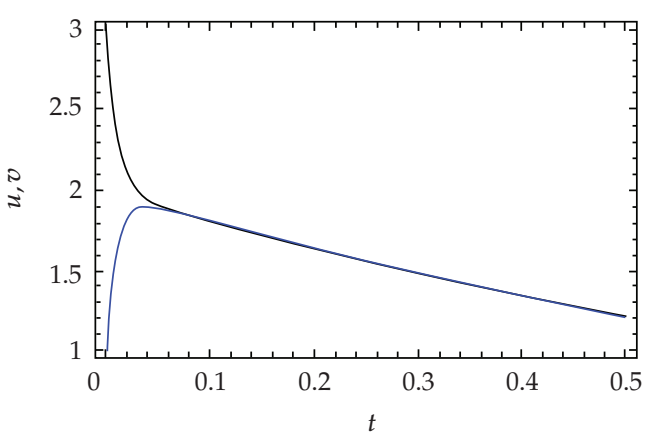

(a)

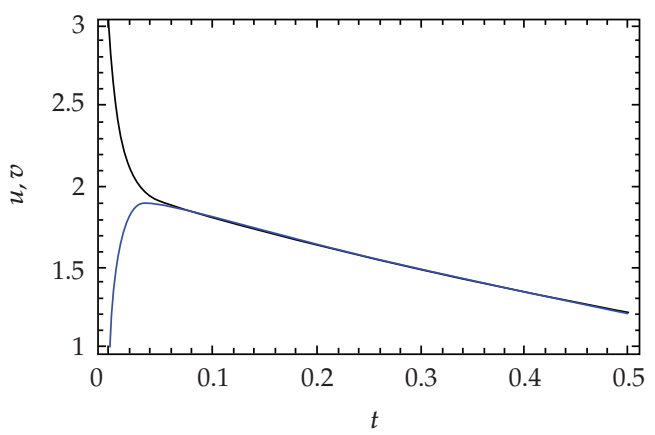

(c)

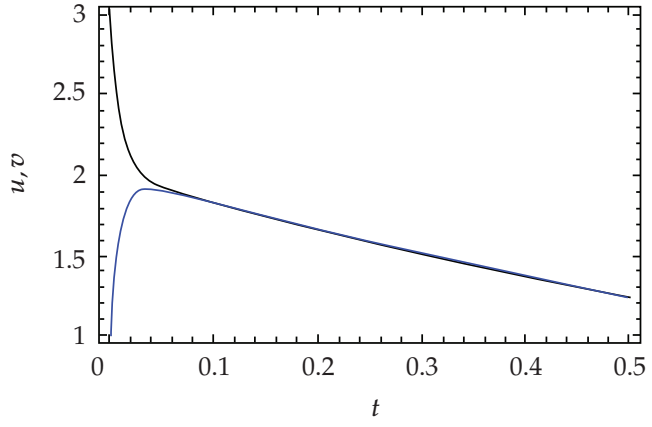

(b)

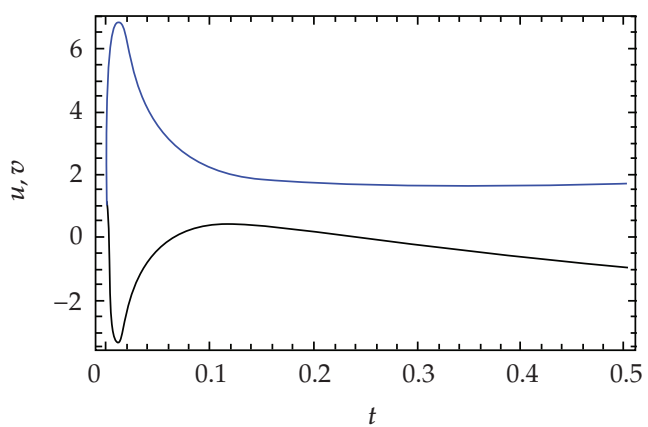

(d)

Figure 1: Solutions of linear stiff system for $k=50, \varepsilon=0.01, \alpha=1$, (a) Exact, (b) Numerical, (c) NHPMPade [10/11], (d) NHPM-Pade [10/11], $k=50, \varepsilon=0.01, \alpha=0.5$ (color figure can be viewed in the online issue).

\section{Concluding Remarks}

The NHPM for solving system of fractional-order differential equations are based on two component procedure and polynomial initial condition. The NHPM applied on fractionalorder Stiff equation, fractional Genesio equation, and the matrix Riccati-type differential equation. The Applications in problems 1-4 are plotted in Figures 1, 2, 3, and 4, which show the accuracy of NHPM. The computations associated with the applications discussed above, were performed by MATHEMATICA. The NHPM is very simple in application and is less computational more accurate in comparison with other mentioned methods. By using this method, the solution can be obtained in bigger interval. Unlike the ADM [19], the NHPM is free from the need to use Adomian polynomials. In this method, we do not need the Lagrange multiplier, correction functional, stationary conditions, and calculating integrals, which eliminate the complications that exist in the VIM [20]. In contrast to the HPM and HAM, in this method, it is not required to solve the functional equations in each iteration. The efficiency of HAM is very much depended on choosing auxiliary parameter $\hbar$. All the applications are taken from [20] with fractional derivatives. 


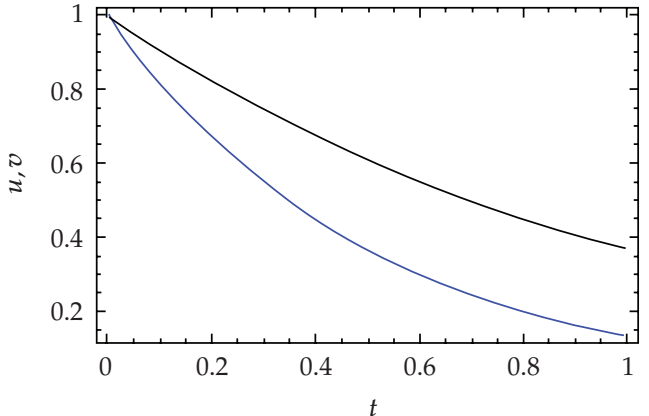

(a)

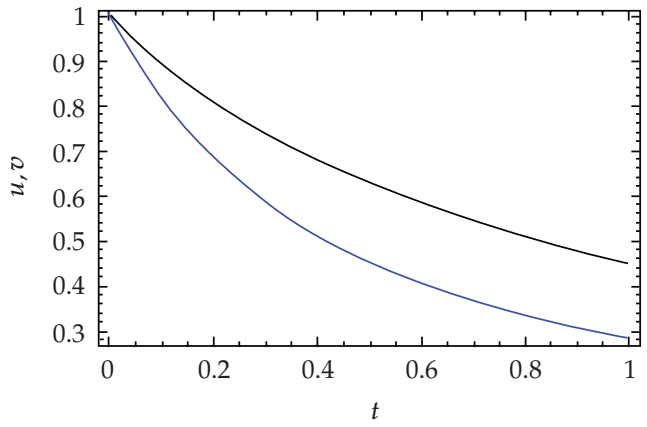

(c)

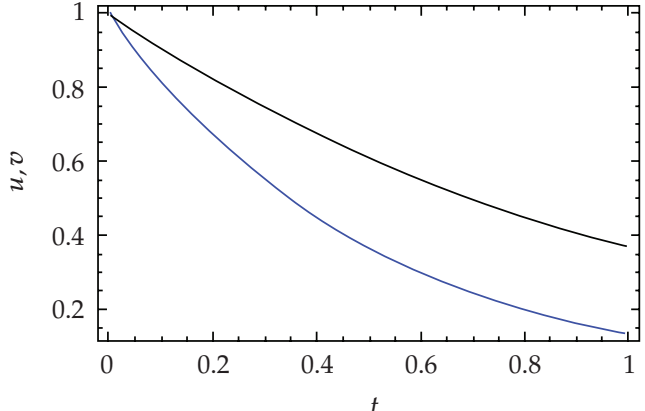

(b)

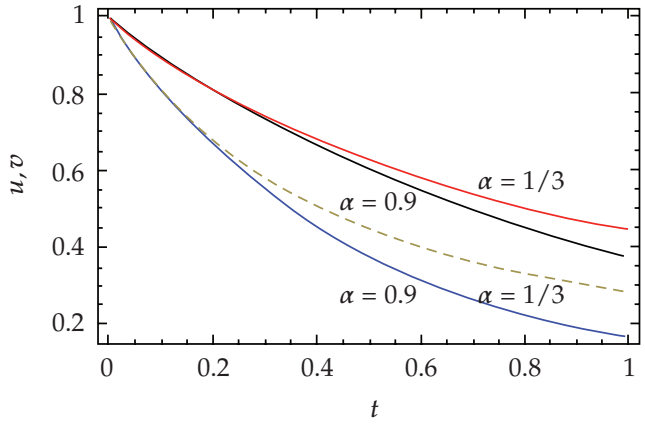

(d)

Figure 2: Solutions of nonlinear stiff system for $\alpha=1$, (a) Exact, (b) Numerical, (c) NHPM, and (c) NHPM, $\alpha=0.9,1 / 3$ (color figure can be viewed in the online issue).

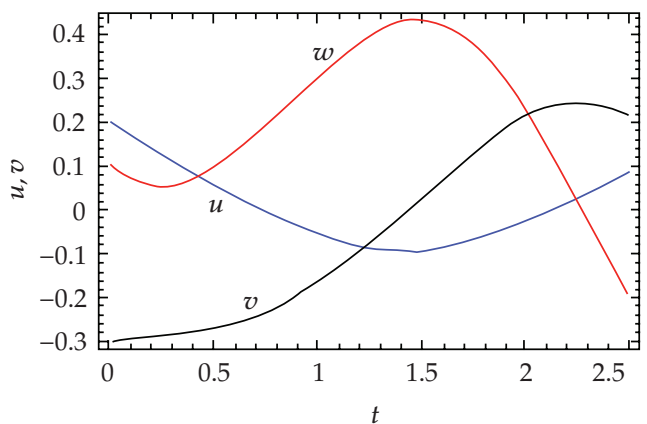

(a)

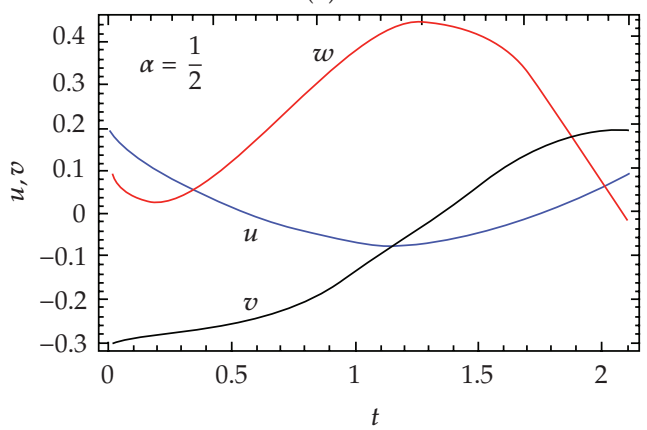

(c)

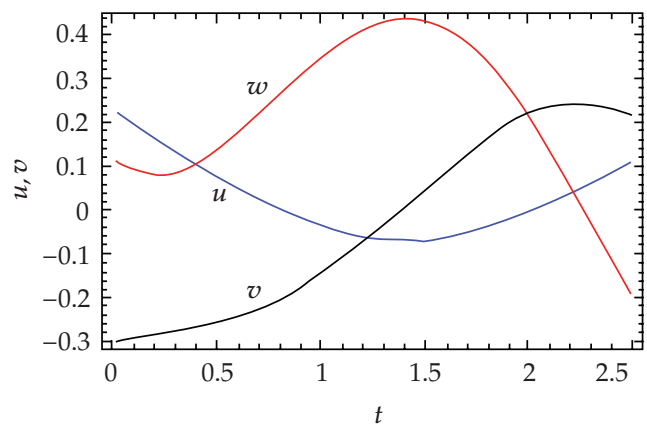

(b)

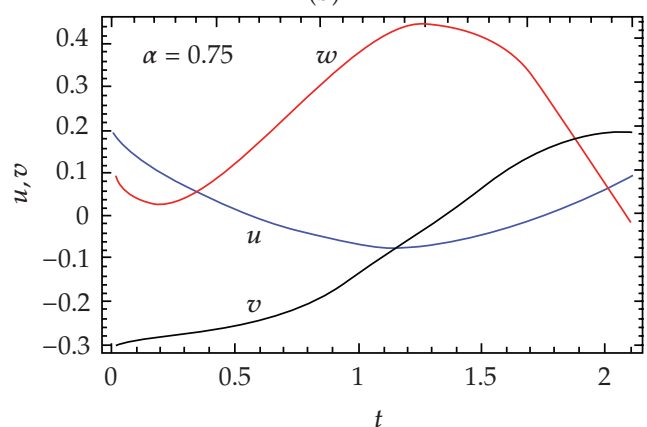

(d)

Figure 3: Solutions of nonlinear Genesio system for (a) Numerical, (b) NHPM $\alpha=1$, (c) NHPM, $\alpha=0.5$, (d) NHPM, $\alpha=0.75$ (color figure can be viewed in the online issue). 


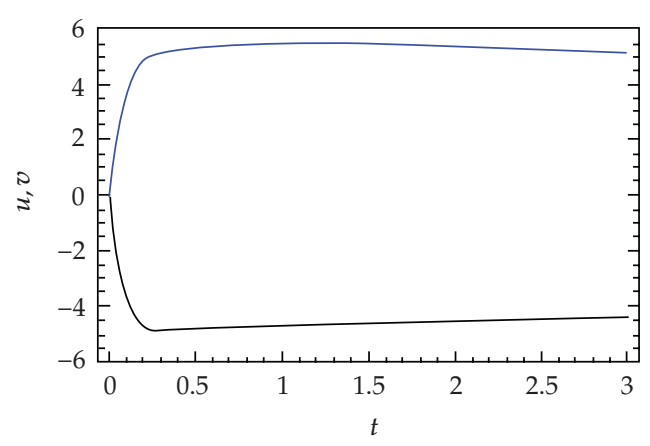

(a)

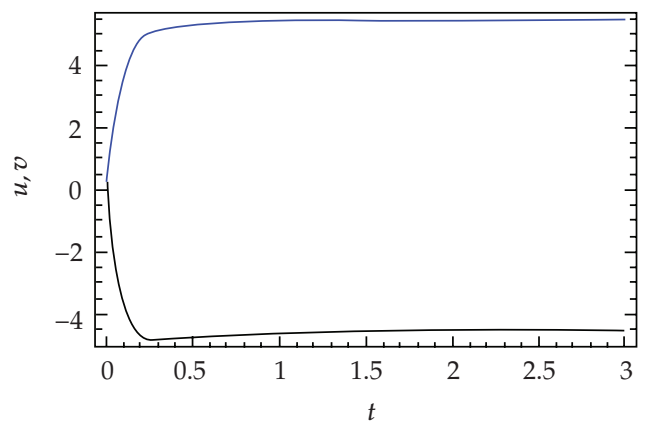

(b)

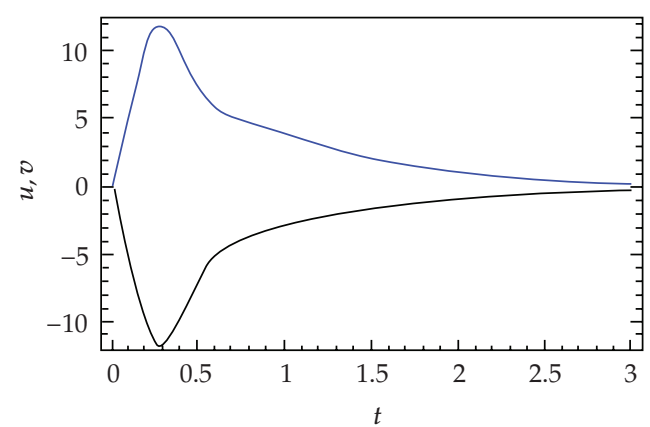

(c)

Figure 4: Solutions of matrix Riccati equations $u=w, v=z$ for $\alpha=1$ (a) Numerical, (b) NHPM-Pade [9/11], (c) NHPM-Pade [9/11], $\alpha=0.5$ (color figure can be viewed in the online issue).

\section{Acknowledgment}

M. Jamil is highly thankful and grateful to the Abdus Salam School of Mathematical Sciences, GC University, Lahore, Pakistan, the Department of Mathematics \& Basic Sciences, NED University of Engineering \& Technology, Karachi-75270, Pakistan, and also the Higher Education Commission of Pakistan for generously supporting and facilitating this research work.

\section{References}

[1] H. M. Kilbas, H. M. Srivastava, and J. J. Trujillo, Theory and Applications of Fractional Differential Equations, Elsevier, Amsterdam, The Netherlands, 2007.

[2] R. L. Bagley and R. A. Calico, "Fractional order state equations for the control of viscoelastically damped structures," Journal of Guidance, Control, and Dynamics, vol. 14, no. 2, pp. 304-311, 1991.

[3] A. Mahmood, S. Parveen, A. Ara, and N. A. Khan, "Exact analytic solutions for the unsteady flow of a non-Newtonian fluid between two cylinders with fractional derivative model," Communications in Nonlinear Science and Numerical Simulation, vol. 14, no. 8, pp. 3309-3319, 2009.

[4] A. Mahmood, C. Fetecau, N. A. Khan, and M. Jamil, "Some exact solutions of the oscillatory motion of a generalized second grade fluid in an annular region of two cylinders," Acta Mechanica Sinica, vol. 26, no. 4, pp. 541-550, 2010.

[5] J. H. He, "Nonlinear oscillation with fractional derivative and its applications," in Proceedings of the International Conference on Vibrating Engineering, pp. 288-291, Dalian, China, 1998. 
[6] N. A. Khan, N.-U. Khan, A. Ara, and M. Jamil, "Approximate analytical solutionsof fractional reaction-diffusion equations," Journal of King Saud University-Science. In press.

[7] J.-H. He, "Homotopy perturbation technique," Computer Methods in Applied Mechanics and Engineering, vol. 178, no. 3-4, pp. 257-262, 1999.

[8] J.-H. He, "A coupling method of a homotopy technique and a perturbation technique for non-linear problems," International Journal of Non-Linear Mechanics, vol. 35, no. 1, pp. 37-43, 2000.

[9] J.-H. He, "Homotopy perturbation method: a new nonlinear analytical technique," Applied Mathematics and Computation, vol. 135, no. 1, pp. 73-79, 2003.

[10] N. A. Khan, A. Ara, and A. Mahmood, "Approximate solution of time-fractional chemical engineering equations: a comparative study," International Journal of Chemical Reactor Engineering, vol. 8, article A19, 2010.

[11] N. A. Khan, N.-U. Khan, M. Ayaz, and A. Mahmood, "Analytical methods for solving the timefractional Swift-Hohenberg (S-H) equation," Computers and Mathematics with Applications. In press.

[12] N. A. Khan, A. Ara, S. A. Ali, and M. Jamil, "Orthognal flow impinging on a wall with suction or blowing," International Journal of Chemical Reactor Engineering. In press.

[13] A. Yıldırım, "Solution of BVPs for fourth-order integro-differential equations by using homotopy perturbation method," Computers E Mathematics with Applications, vol. 56, no. 12, pp. 3175-3180, 2008.

[14] H. Koçak, T. Öziş, and A. Yıldırım, "Homotopy perturbation method for the nonlinear dispersive $\mathrm{K}(\mathrm{m}, \mathrm{n}, 1)$ equations with fractional time derivatives," International Journal of Numerical Methods for Heat \& Fluid Flow, vol. 20, no. 2, pp. 174-185, 2010.

[15] Y. Khan, N. Faraz, A. Yildirim, and Q. Wu, "A series solution of the long porous slider," Tribology Transactions, vol. 54, no. 2, pp. 187-191, 2011.

[16] N. A. Khan, A. Ara, S. A. Ali, and A. Mahmood, "Analytical study of Navier-Stokes equation with fractional orders using He's homotopy perturbation and variational iteration methods," International Journal of Nonlinear Sciences and Numerical Simulation, vol. 10, no. 9, pp. 1127-1134, 2009.

[17] H. Aminikhah and J. Biazar, "A new HPM for ordinary differential equations," Numerical Methods for Partial Differential Equations, vol. 26, no. 2, pp. 480-489, 2010.

[18] H. Aminikhah and M. Hemmatnezhad, "An efficient method for quadratic Riccati differential equation," Communications in Nonlinear Science and Numerical Simulation, vol. 15, no. 4, pp. 835-839, 2010.

[19] Y. Khan and N. Faraz, "Modified fractional decomposition method having integral w.r.t $(d \xi)^{\alpha}$," Journal of King Saud University-Science. In press.

[20] A. S. Bataineh, M. S. M. Noorani, and I. Hashim, "Solving systems of ODEs by homotopy analysis method," Communications in Nonlinear Science and Numerical Simulation, vol. 13, no. 10, pp. 2060-2070, 2008. 